\title{
Communication Signal Source Development in Contactless IC Card
}

\author{
LIU Xinyu \\ School of Communication \& Information Engineering \\ Beijing Information Science \& Technology University \\ Beijing, P.R. China, cell phone: 86-15210968358 \\ marshal.liuxinyu@yahoo.com.cn
}

\author{
LIN Mingquan \\ Institute of astronomy \\ China Academic of Science \\ Beijing, P.R. China
}

\begin{abstract}
Contactless IC card is a new technology in IC field. It is the combination of radio frequency identification and IC. Especially, in bank system, magnetic stripe cards will give place to IC smart card in the near future. Therefore, an increasing number of contactless IC cards will be required to test and certify. In this paper, we have studied the contactless IC card communication system and communication protocol principle of Chinese financial integrated circuit (IC) card specification. According to the ISO14443 Type A and Type B protocol, we design contactless IC card communication signal source module which is based on the RF transceiver chip PN512. The function of the signal source is to send awakening signal to the contactless IC. We simulate a contactless card Reader, output the wireless signal in form of wired signal. Finally, a more precise way is chosen from alternative options to produce a standard contactless IC card communication signal.
\end{abstract}

Keywords-component; Contactless IC card; Signal source; ARM7; PN512 RF chip;

\section{INTRODUCTION}

IC smart cards are becoming increasingly prevalent in our lives. The mainstream in the contactless IC smart RF card field is Philips Corporation's Mifare technology, which has been set as the international standards of IEC 14443 TYPEA. Some large European manufacturers of the IC card reader and the IC card software design companies use Mifare technology as a standard to develop and promote the IC card industry.

At present, the typical contactless IC card property test needs manual control to send polling instruction and is relatively cumbersome and its frame work chart is shown in Fig 1. Furthermore, given that the signal acquisition facility is to collect wireless signal, the accuracy of the data needs to be enhanced. In this paper, the purpose of our task is to integrate PC, READER and signal acquisition unite on a circuit board of communication signal source. Then the test tool can be simplified and the test efficiency can be improved. The frame work chart is shown in Fig 2. We also designed three signal transmission circuit schemes: sending signal by antenna, transformer, and wire. Then the results of the three schemes can be evaluated. At last, the chosen signal would access the adjustable power amplifier

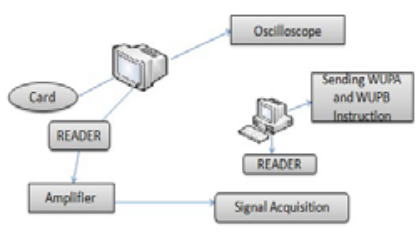

Fig. 1 The current contactless IC card electric property test system

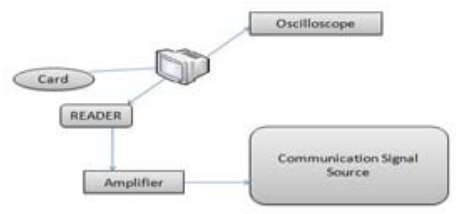

Fig. 2 The improved contactless IC card electric property test system

\section{THE ENTIRETY FRAMEWORK}

To differentiate from convention, the method gives an efficient way to process signals with high degree of accuracy. Besides, the test tool can be simplified and the test efficiency will be enhanced. The entirety design is shown in Fig. 3.

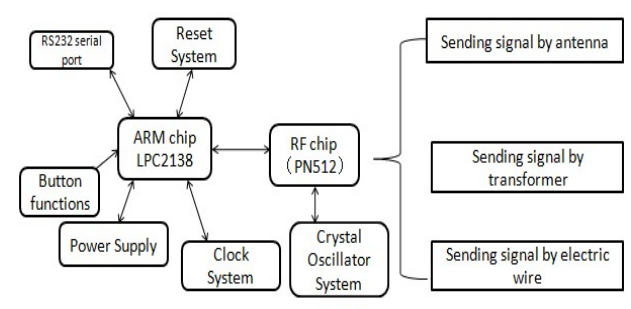

Fig. 3 The design of framework

The ARM module adopts LPC2138 microprocessor (a product of PHILIPS company), which is equipped ARM7TDMI-S core and LQFP64 package. With Small package and very low power consumption, LPC2138 is suitable for our signal source design. It also has two channels of UART, 32KB on-chip static RAM and 512KB on-chip FLASH program memory. 
PN512 chip (a product of NXP) is used in RF module, which supports both Type A and Type B coding. PN512 chip is a product for the $13.56 \mathrm{MHz}$ contactless communication with high integration transceiver IC.

\section{DESIGN AND IMPLEMENTATION}

\section{A. ARM module design}

1) Power supply circuit

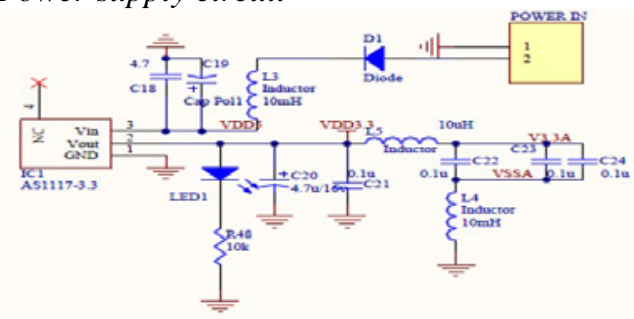

Fig. 4 Power supply circuit diagram

In the system, LPC2138 needs power supply and the I/O port's power supply quota is $3.3 \mathrm{~V}$. Considering the range of RF module's AVDD power and DVDD is $2.5 \mathrm{~V}$ to $3.6 \mathrm{~V}$, or $1.6 \mathrm{~V}$ to $3.6 \mathrm{~V}$, we select $3.3 \mathrm{~V}$ power supply for the circuit. The power supply circuit diagram can be seen in Fig.4. The 5V DC current goes out through the adapter, and its conduction direction can be specified with the D1 diode. After being filtered by C18 and C19, the current can be stabilized to 3.3V by going through AS1117-3.3 chip. LPC2138 has independent analog power pin V3A and VSSA. In order to reduce noise and error probability, we should isolate AVDD and DVDD, L4 and L5 are the components for power isolation (to isolate high frequency noise in DVDD). AS1117-3.3 chip is a positive low dropout regulator and its voltage drop is $1.2 \mathrm{~V}$ at $1 \mathrm{~A}$. The AMS1117 integrated internal overheating protection and current limiting circuit is an ideal choice for battery-power.

2) Reset circuit

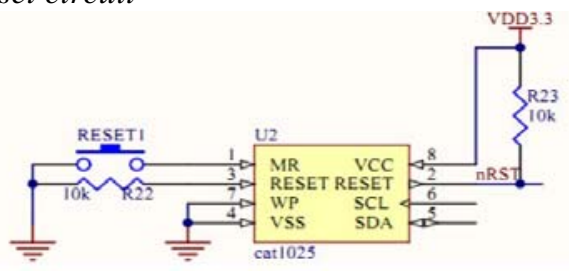

Fig. 5 reset circuit diagram

Due to the ARM chip's property of high speed, low power consumption, its working voltage noise tolerance is low, the power supply ripple, transient response performance, clock source stability, power monitoring reliability etc, it also needs higher request for design. In the reset circuit diagram (which is shown in Fig.4), signal nRST is connected to the RESET pin of LPC2138 chip. When the reset button RST is pressed, the RESET pin in cat1025 would immediately output RESET signal. Consequently, the LPC2138 chip could reset. Moreover, two RESET pins respectively connect to a drop-up resistance and drop-down one.

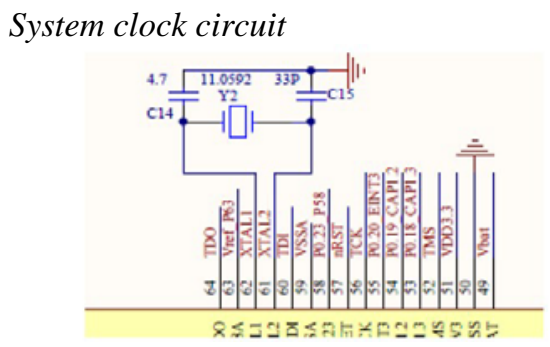

Fig. 6 system clock circuit diagram

LPC2100 series ARM7 micro controller can use external crystal oscillator or external clock source, its internal PLL circuit can make the system run faster (CPU maximum operating clock to $60 \mathrm{MHZ}$ ) by adjusting system clock. Without the PLL function and ISP download function, the external crystal oscillator frequency range is $1 \mathrm{MHZ} \sim 30$ MHZ, the external clock frequency range is $1 \mathrm{MHZ} \sim 50 \mathrm{MHZ}$; Otherwise the external crystal oscillator frequency range is 10 MHZ $25 \mathrm{MHZ}$, external clock frequency range is $10 \mathrm{MHZ}$ $25 \mathrm{MHZ}$. This circuit adopts the external $11.0592 \mathrm{MHz}$ crystal oscillator, circuit diagram is as shown in Fig. 6. With 11.0592 $\mathrm{MHz}$ crystal vibration, the serial interface baud rate's accuracy could be enhanced. At the same time, it is able to support LPC2138 microcontroller chip's internal PLL function and ISP function.

\section{Communication interface}

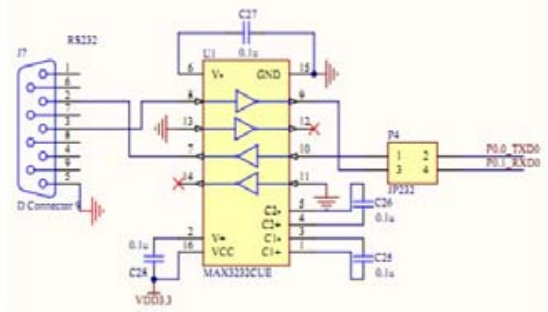

Fig. 7 communication interface circuit diagram

The ARM chip and RF chip use SPI bus to transmit. When ISP (in system programming), JP232 connector needs to be short-circuited, in order to detect the low level P0.14 pin when the CPU is on reset. MAX323CUE chip is chosen in RS232 interface to realize the transformation of two levels. As can be seen in Fig. 7, RX (receiving data line), TX (sending number) and GND (signal wire) in RS232 interface is used to accomplish communication transmission. To avoid the condition that the ISP software does not recognize ARM chip, the RS232 interface has to be connected to ARM URAT0.

\section{B. RF interface module}

The RF part is consisted of RF chip, power filtering, analog signal filtering and peripheral circuits and it is the central part in the card reader circuit, which plays the role of the bridge between CPU and RFID. There are three schemes in the antenna design. They are respectively acquire directly output signal from card read chip, to acquire signal after the pin signal goes through a 1:1 transformer and to acquire signal through wireless antenna. Our work is to observe which of the plans signal is more accurate. 
1) To acquire signal through antenna

To provide enough power supply to the RFID card, the minimum coupling coefficient between antenna and card is 0.3 . The inductance of antenna coil is:

$$
\mathrm{L}=2 * I * \ln (1 / \mathrm{D}) * \mathrm{~N}^{1} .8
$$

(L: antenna inductance, I: length of ring conductor, D: width of conductor, $\mathrm{N}$ : coil turns)

\section{2) To acquire signal through transformer}

The fundamental functions of transformer and antenna in the first plan are identical, except that transformer could turn wireless signal into wire signal.

3) To acquire output signal directly by wire

This method is mainly used to compare with the two other methods above.

\section{Software development}

The software in the system is developed in the ADS1.2 environment.

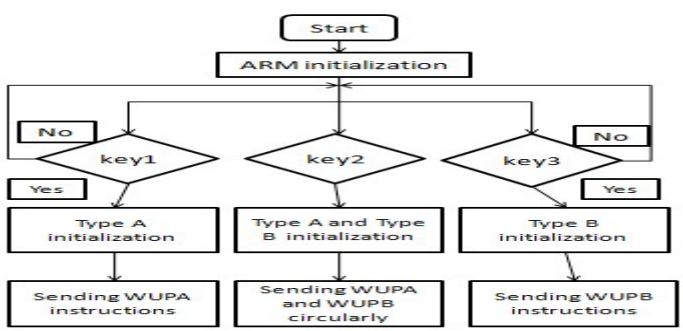

Fig. 8 software framework

As is shown in Fig.8, system initialization includes ARM register, SPI, RF chip initialization. After initialization, it would check the three keys' statuses. When the A key is pressed, interruption will be generated, the system will configure PN512 chip Type A and Type B relevant register through the SPI bus. Otherwise it will circularly send WUPA and WUPB instructions.

\section{EXPERIMENTAL RESUTLS}

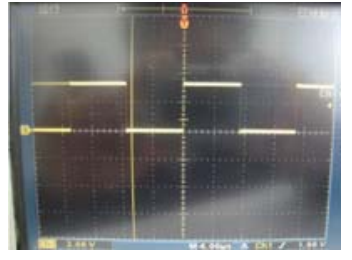

Fig. 9 (a) SPI bus signal

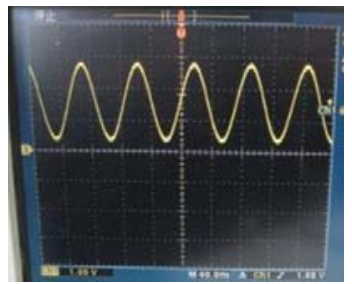

Fig. 9 (c) $13.56 \mathrm{MHz}$ carrier Fig. 9 (d) Type A 100\% ASK modulation
We compile and run the software with ADS1.2, download it in the hardware through ISP. The results are as following: clock signal in ARM, namely SPI bus clock signal is shown in Fig. 9(a), which indicates that the crystal oscillator works successfully. MISO pin signal is displayed in Fig. 9 (b), which is the signal from ARM to PN512 chip to configure the relevant register. The configured PN512 register output 13.56MHz (displayed in Fig. 9 (c)), which is a precondition for the future signal modulation. Type A signal waveform is shown in Fig. 9 (d). According to the WUPA frame format, ARM send 0x52 (awaking instruction) to RF chip by SPI bus.

\section{CONCLUSION}

The paper presents an improved contactless IC card communication signal source design which is based on ISO/IEC14443 standard. PC, READER and signal acquisition unite are integrated on a circuit board of communication signal source and the test tool can be simplified and the test efficiency can be improved. By using ISP and JTAG to download software, we accomplished the communication between ARM and RF chip. Besides, we also realized the configuration of registers of Type A and Type B, WUPA frame instructions sending, sending data with antenna or transformer by PN512's modulation.

However, the stability of the system has to be enhanced. In order to make our design to a real product, we should integrate amplifier module, monitor module and etc. on a single PCB board. At last, the current system does not involve operate system due to meet our basic needs. So we should improve the development performance of our system in the future.

\section{REFERENCES}

[1] XU Xiangming, Altium designer quick start [M].

[2] Union-pay integrated circuit card specifications, part 6, contactless IC card [M].

[3] WANG Aiying, Smart card technology: IC card and RFID tag [M].

[4] ISO/IEC. Identification cards-Contactless integrated circuit(s) cards Proximity card-part 1: Physical characteristics[S]. ISO/IEC14443-1, 2001.

[5] ISO/IEC. Identification cards-Contactless integrated circuit(s) cards Proximity card-part 3: Initialisation and Anticollision [S]. ISO/IEC14443-2, 2001.

[6] YAN Shengli, Altium designer skills: schematic diagram and PCB design [M].

[7] HAN Shan, GUO Yun, FU Haiyan, ARM Microprocessor application development technical explanation and example analysis [M](2007).

[8] ZHOU Ligong, ARM embedded system experimental course (3) extended experiment [M].

[9] WANG Kaiyuan, The contactless IC card reader design based on the ARM [D].

[10] ZHOU Ligong, Zhang Hua, Engineer of ARM7 LPC 213 x_214x, part 1, [M].

[11] WANG Kaiyuan, The contactless IC card reader design on the ARM [D](2009). 